Proyecciones Journal of Mathematics

Vol. 34, No 3, pp. 219-228, September 2015.

Universidad Católica del Norte

Antofagasta - Chile

\title{
Holomorphically proyective Killing fields with vectorial fields associated in kahlerian manifolds
}

\author{
Richard Malavé Guzmán \\ U. Politécnica Territorial del oeste de Sucre C. R., Venezuela \\ Franmary López \\ U. Politécnica Territorial del oeste de Sucre C. R., Venezuela \\ and \\ Rodrigo Martinez \\ Universidad de Oriente, Venezuela \\ Received : October 2013. Accepted : April 2015
}

\begin{abstract}
Taking into account the harmonic and scalar curvatures in the study of Killing transformations between spacial complex (Einstenian, Peterson-Codazzi, Recurrent) and kaehlerian $M$ spaces with almost complex J structure, we prove that there exists an holomorphically proyective transformation between $M$ spaces and complex spaces.
\end{abstract}

Keywords: Holomorphically proyective, Killing fields, kaehlerian manifolds.

MSC 2010: 53A20, 53B35,53C15. 


\section{Introduction and preliminaries}

By the end of the $20^{\text {th }}$ century researchers started to link the concept of proyectivity with the phenomenon of complex manifolds specially in terms of their hollomorphic properties. Then, kaehlerian and hermithian manifolds as well as complex hyper surfaces and other manifolds were considered embedded into special transformations. At this point a vast number of publications arose in relation with the concepts of compact K manifolds, proyective infinitesimal transformations in Riemmanian manifolds with additive curvature properties and hollomorphic proyective equivalences and others. Based on [1] [2] and [3], this research studies Kaehler holomorphically proyective manifolds with almost complex structures by using the geometric properties of the harmonic and scalar curvatures evaluated over Killing vectorial fields. Two important applications result from this, the Einstenian and the constant curvature spaces.

Considering $(M, g, J)$ as a kaehlerian manifold of $2 n \geq 4$ dimension with a $g=\left(g_{i j}\right)$ Riemannian metric and an almost-complex structure $J=$ $J_{i j}$ where $J_{i j}=-J_{j i}$ and with a Riemannian curvature tensor of $R_{k j i}^{h}=$ $\partial_{k} \Gamma_{j i}^{h}-\partial_{j} \Gamma_{k i}^{h}+\Gamma_{k a}^{h} \Gamma_{j i}^{a}-\Gamma_{j a}^{h} \Gamma_{k i}^{a}$ the $S_{j i}=R_{a j i}^{a}$ then the Ricci tensor and the $r=g^{b a} S_{b a}$ scalar curvature satisfy the following proprieties:

$$
\begin{array}{ccc}
\text { i) } S_{j i}=J_{i}^{b} J_{i}^{a} S_{b a} & \text { ii) } H_{j i}=J_{j}^{a} S_{a i} & \text { iii) } H_{j i}+H_{i j}=0 \\
i v) H_{j i}=J_{i}^{b} J_{i}^{a} H_{b a} & \text { v) } J_{i}^{a} J_{a}^{h}=-\delta_{i}^{h} & \text { vi) } J_{j}^{a}=-i \delta_{j}^{a} \\
\text { vii) } g_{i j}=J_{i}^{a} J_{j}^{b} g_{a b} & \text { viii) } \nabla_{j} \widetilde{F}_{i}=-\nabla_{i} \widetilde{F}_{j} & i x) \widetilde{F}_{i}:=J_{i}{ }^{a} F_{a}
\end{array}
$$

where

$\mathrm{J}^{j i}=g^{j a} J_{a}{ }^{i}, H_{j i}=J_{a}{ }^{i} S_{a i}$. The Lie operator derivative in the vectorial field direction $X$ for $R_{k j i}{ }^{h}$ and ${ }_{h j i}$ is represented respectively by,

$$
L_{X} R_{k j i}^{h}=\nabla_{k} L_{X} \Gamma_{j i}^{h}-\nabla_{j} L_{X} \Gamma_{k i}^{h} y L_{X} \Gamma_{j i}^{h}=\nabla_{j} \nabla_{i} X^{h}+R_{a j i}^{h} X^{a} .
$$

If $X$ is a vectorial field then

i) $X$ is a Killing field if satisfies

$$
L_{X} g_{j i}=0, \forall i, j=\overline{i, n} .
$$

ii) $X$ is an holomorphically projective transformation when

$$
L_{X} \Gamma_{j i}^{h}=\delta_{i}^{h} F_{i}+\delta_{j}^{h} F_{i}-J_{j}^{h} J_{i}^{a} F_{a}-J_{i}^{h} J_{j}^{a} F_{a},
$$


where $F=\left(F^{i}\right)$ is a particular vector associated to $X$.

Two metric $g=\left(g_{i j}\right)$ y $\bar{g}=\left(\bar{g}_{i j}\right)$ defined on $M$, they are hollomorphic proyective equivalences if

$$
\bar{\Gamma}_{k i}^{k}=\Gamma_{j i}^{k}+F_{i} \delta_{i}^{k}+F_{j} \delta_{i}^{k}-J_{j}^{k} \widetilde{F}_{i}-J_{i}^{k} \widetilde{F}_{j},
$$

where $\widetilde{F}_{i}=J_{i}^{a} F_{a}$.

Tensors for harmonic and scalar curvatures are defined on the manifold $M$ by means of the following relations:

$$
\begin{gathered}
\nabla_{a} R_{k j i}{ }^{a}=\nabla_{k} S_{j i}-\nabla_{j} S_{k i}, \\
R=g^{b a} S_{b a}
\end{gathered}
$$

respectively where $S_{j i}=R_{a j i}{ }^{a}$ is the tensor Ricci. The Laplacian of $f$ is defined by

$$
\Delta f=\nabla^{a} \nabla_{a} f=\Delta f,
$$

where $f=\frac{1}{n+2} \nabla_{a} X^{a}$ with $f \in C^{\infty}(M)$ and $F_{j}=\nabla_{j} f$.

The classic commutative relationship of $L_{X}$ and de $\nabla$ for a tensor $Y$ of $(1,2)$ type is given by

$L_{X} \nabla_{k} Y_{j i}{ }^{h}-\nabla_{k} L_{X} Y_{j i}^{h}=\left(L_{X} \Gamma_{k a}^{h}\right) Y_{j i}^{h}-\left(L_{X} \Gamma_{k j}^{h}\right) Y_{a i}^{h}-\left(L_{X} \Gamma_{k j}^{a}\right) Y_{a i}^{h}-\left(L_{X} \Gamma_{k i}^{a}\right) Y_{j i}{ }^{h}$.

Being $X$ an holomorphically proyective transformation with an $F$ associated vector then the following identities are satisfied, watch [1]

i $2 S_{i}^{a} F_{a}=-\nabla_{i}(\Delta f)$

ii $\nabla_{j} F_{i}=J_{i}^{a} J_{j}^{b} \nabla_{b} F_{a}$

iii $\nabla_{k} \nabla_{j} F_{i}=-J_{k}{ }^{b} J_{j}^{a} R_{i a b}^{c} F_{c}$.

In [3] proof

$$
S_{i j}=\bar{S}_{i j}+\tau\left(F_{i j}-\bar{F}_{j i}\right)
$$

where $F_{i j}=\nabla_{i} \nabla_{j} f, \tau-$ parameter.

An $A_{n}=(M, \nabla)$ space is a Peterson Codazzi one if $\nabla_{k} S_{j i}=\nabla_{j} S_{k i}$. If $\nabla_{l} R_{i j k}^{h}=F_{l} R_{i j k}^{h}$ it is a recurrent space where $F_{l} \neq 0$ or it is an Einstein space if $S=\lambda g$ taking $S$ as the Ricci and $g$ an the metric tensors and $\lambda$ as a parameter. 
Lemma 1.1. If $M$ is a compact Kaehlerian manifold of dimension $n$ with a scalar curvature $R$ and it admits an holomorphically proyective transformation then the following equations are fulfilled,

i) $\Delta f=-\frac{2 R}{n} f$

ii) $S_{i}{ }^{a} F_{a}=\frac{R}{n} F_{i}$.

Proof. i) Since $A_{n}$ is a recurrent space and $M$ admits an holomorphically proyective transformation then we obtain

(1.4) $g^{h i} \nabla_{b} \nabla_{j} X_{i}+g^{h i} R_{a b j i} X^{a}-F_{b} \delta_{j}^{h}-F_{j} \delta_{b}^{h}+F_{a} J_{b}^{a} J_{j}^{h}+F_{a} J_{j}^{a} J_{b}^{h}=0$,

multiplying (1.4) by $g_{h k}$ and applying $\nabla^{b}$ it results that

$$
\nabla^{b}\left(\nabla_{b} \nabla_{j} X_{i}+R_{a b j i} X^{a}-F_{b} g_{j i}-F_{j} g_{b i}+F_{a} J_{b}^{a} J_{j i}+F_{a} J_{j}^{a} J_{b i}\right)=0 .
$$

Now using Ricci's and Bianchi's identities we obtain

$$
\left(R_{a b j i}-2 R_{b j i a}\right) \nabla^{b} X^{a}-R_{a i} \nabla_{j} X^{a}+R_{j}^{a} \nabla_{a} X_{i}-\left(\nabla_{a} R_{j i}\right) X^{a}=0,
$$

finally by applying $\nabla^{j}$ the result is

$$
\begin{aligned}
-2 \nabla_{i} R_{b a} \nabla^{b} X^{a} & =0 \Rightarrow \nabla_{i} R_{b a} L_{X} g^{b a}=0 \\
& \Rightarrow-2 R F_{i}=n \nabla_{i}(\Delta f) \\
& \Rightarrow \nabla_{i}(\Delta f)=-\frac{2 R}{n} f=\nabla_{i}\left(-\frac{2 R}{n} f\right) \\
& \Rightarrow \Delta f=-\frac{2 R}{n} f
\end{aligned}
$$

due to $(n \Delta f+2 R f)$ is constant for being

$$
\int_{M} \Delta f d \sigma=\int_{M} f d \sigma=0
$$

a compact $M$ and $d \sigma$ is a volumetric element of $M$. Finally we conclude that

$$
\Delta f=-\frac{2 R}{n} f
$$

ii) The demonstration is obtained by using I and part (i) from this lema.

Lemma 1.2. Let $X$ be an holomorphically proyective transformation with an $F$ associated vector then

$$
L_{X} S_{j i}=-(n+2) \nabla_{j} F_{i}
$$


Proof. Using the definition of $L_{X} R_{k j i}^{h}$ we have

$$
L_{X} S_{j i}=L_{X} R_{h j i}{ }^{h}=\nabla_{h} L_{X} \Gamma_{j i}^{h}-\nabla_{j} L_{X} \Gamma_{h i}{ }^{h},
$$

since $X$ is an holomorphically projective transformation then

$$
\begin{aligned}
L_{X} S_{j i}= & \nabla_{j} F_{i}+\nabla_{i} F_{j}-J_{j}^{h} J_{i}^{a} F_{a}-J_{i}^{h} J_{j}^{a} F_{a}-n \nabla_{j} F_{i}-\nabla_{j} F_{i}+ \\
& +i n J_{i}^{a} \nabla_{j} F_{a}-\nabla_{j} F_{i} .
\end{aligned}
$$

By considering the real part we obtain the desired result

$$
L_{X} S_{j i}=-n \nabla_{j} F_{i}-2 \nabla_{j} F_{i}=-(n+2) \nabla_{j} F_{i} .
$$

\section{Results}

The following theorem allows a Kaehlerian space to become into a PetersonCodazzi space under the hypothesis that the former is holomorphically projective.

Theorem 2.1. Let $M$ be a Kaehlerian manifold and $X$ be an holomorphically projective killing field with an associated vectorial field $F$ then

$$
\begin{gathered}
L_{X}\left(\nabla_{j} S_{k i}-\nabla_{k} S_{j i}\right) \\
=\left\{(n+2) R_{j k i}^{a}-S_{k i} \delta_{j}^{a}+S_{j i} \delta_{k}^{a}-J_{i}^{a} H_{k i}+J_{k}^{a} H_{j i}+2 J_{i}^{a} H_{j k}\right\} F_{a} .
\end{gathered}
$$

Proof. Using the classic relation of commutation for a $(0,2)$ type tensor we obtain that

$$
\left(L_{X} \nabla_{j} S_{k i}-L_{X} \nabla_{k} S_{j i}\right)-\left(\nabla_{j} L_{X} S_{k i}-\nabla_{k} L_{X} S_{j i}\right)=\left(L_{X} \Gamma_{k i}^{a}\right) S_{j a}-\left(L_{X} \Gamma_{j i}^{a}\right) S_{k a} .
$$

If by hypothesis we consider $X$ as an holomorphically projective transformation by using (1.2) then we have that

$$
L_{X} \Gamma_{j i}{ }^{a}=\delta_{j}^{a} F_{i}+\delta_{i}^{a} F_{j}-J_{j}^{a} J_{i}^{h} F_{h}-J_{i}^{a} J_{j}^{h} F_{h} .
$$

Furthermore according to lemma (1.2),

$$
L_{X} S_{j i}=-(n+2) \nabla_{j} F_{i}
$$


and analogically we obtain $L_{X} \Gamma_{k i}^{a}$ and $L_{X} S_{k i}$.

By Substituting (2.3) and (2.4) in (2.2) it results

$$
\begin{gathered}
\left(L_{X} \nabla_{j} S_{k i}-L_{X} \nabla_{k} S_{j i}\right)-\left(\nabla_{j}\left[-(n+2) \nabla_{k} F_{i}\right]-\nabla_{k}\left[-(n+2) \nabla_{j} F_{i}\right]\right) \\
=\left(\delta_{k}^{a} F_{i}+\delta_{i}^{a} F_{k}-J_{k}^{a} J_{i}^{h} F_{h}-J_{i}^{a} J_{k}^{h} F_{h}\right) S_{j a} \\
-\left(\delta_{j}^{a} F_{i}+\delta_{i}^{a} F_{j}-J_{j}^{a} J_{i}^{h} F_{h}-J_{i}^{a} J_{j}^{h} F_{h}\right) S_{k a} .
\end{gathered}
$$

By doing certain manipulations and using simplification we conclude that

$$
\begin{gathered}
\left\{(n+2) R_{j k i}{ }^{a}-S_{k i} \delta_{j}^{a}+S_{j i} \delta_{k}^{a}-J_{i}^{a} H_{k i}+J_{k}^{a} H_{j i}+2 J_{i}^{a} H_{j k}\right\} F_{a} \\
=L_{X}\left(\nabla_{j} S_{k i}-\nabla_{k} S_{j i}\right) .
\end{gathered}
$$

From here on some applications of the previous results will be given.

1) If $\nabla_{j} S_{k i}=\nabla_{k} S_{j i}$ then $M$ is Kaehler-Peterson-Codazzi space and

$$
\left\{(n+2) R_{j k i}^{a}-S_{k i} \delta_{j}^{a}+S_{j i} \delta_{k}^{a}-J_{i}^{a} H_{k i}+J_{k}^{a} H_{j i}+2 J_{i}^{a} H_{j k}\right\} F_{a}=0 .
$$

\section{Consequence i}

A Kaehler-Peterson-Codazzi space has an harmonic curvature since,

$$
\nabla_{j} S_{k i}=\nabla_{k} S_{j i} \Leftrightarrow \nabla_{a} R_{j k i}^{a}=0 .
$$

\section{Consequence ii}

A Kaehler-Peterson-Codazzi space is an Einstenian space if the former has a constant scalar curvature. Factually by applying $g^{k i}$ into (2.5) results in

$$
\left\{(n+2) g^{k i} R_{j k i}{ }^{a}-R \delta_{j}^{a}+g^{a i} S_{j i}-J_{i}^{a} g^{k i} H_{k i}+J_{k}^{a} g^{k i} H_{j i}+2 J_{i}^{a} g^{k i} H_{j k}\right\} F_{a}=0 .
$$

Since $F_{a} \neq 0$ and developing the three last terms we have,

$$
(n+2) g^{k i} R_{j k i}{ }^{a}-R \delta_{j}^{a}+g^{a i} S_{j i}-J_{j}^{a} J_{k}^{b} g^{k i} S_{b i}+3 J_{k}^{a} J_{j}^{b} g^{k i} S_{b i}=0,
$$

by making the contraction $a=j$ and adding up from 1 to $n$ we obtain

$$
g_{k i}(n R+2 R-n R+R)=3 S_{k i},
$$


In this way we conclude that $S_{k i}=\frac{R}{n} g_{k i}$. In other words the KaehlerPeterson-Codazzi space is an Einstenian space.

2) If $M$ is a recurrent space then

$$
(n+2) R_{j k i}^{a} F_{a}-L_{X}\left(R_{j k i}^{a} F_{a}\right)=S_{k i} \delta_{j}^{a}-S_{j i} \delta_{k}^{a}+J_{j}^{a} H_{k i}-J_{k}^{a} H_{j i}-2 J_{i}^{a} H_{j k} F_{a} .
$$

\section{Consequence}

If $M$ is an harmonic curvature and $W=\left\{F=\left(F^{i}\right): F \neq 0\right\}$ with

$$
F_{j} F^{k}=\left\{\begin{array}{ccc}
\|F\|^{2} & \text { si } & j=k \\
0 & \text { si } & k \neq j
\end{array}\right.
$$

then $M$ has a null scalar curvature.

As a matter of fact if $M$ admits an harmonic curvature then making the contraction $l=a$ and summing up from 1 to $n$ in the relation

$$
\nabla_{l} R_{j k i}{ }^{a}=R_{j k i}{ }^{a} F_{l},
$$

we obtain,

$$
\nabla_{l} R_{j k i}{ }^{a}=R_{j k i}{ }^{a} F_{l} \Rightarrow R_{j k i}{ }^{a}=0 .
$$

from (2.1) we obtain

$$
S_{k i} F_{j}-S_{j i} F_{k}+H_{k i} \widetilde{F}_{j}-H_{j i} \widetilde{F}_{k}-2 H_{j k} \widetilde{F}_{i}=0
$$

and multiplying the previous relation by $g^{k i}$ it results that

$$
g^{k i} S_{j i} F_{k}-g^{k i} S_{k i} F_{j}+g^{k i} H_{j i} \widetilde{F}_{k}-g^{k i} H_{k i} \widetilde{F}_{j}+2 g^{k i} H_{k j} \widetilde{F}_{i}=0 .
$$

Therefore

$$
S_{j}^{k} F_{k}-r F_{j}=H_{j}^{k} \widetilde{F}_{k},
$$

wherein by applying $F^{j}$ it results that $r=0$. This way we conclude that the manifold is plain.

\section{Example}

Be Einstein compact Kaehlerian spaces $A_{n}=(M, \nabla)$ and $\bar{A}_{n}=(M, \bar{\nabla})$, with metric $g=\left(g_{i j}\right)$ y $\bar{g}=\left(\bar{g}_{i j}\right)$ hollomorphic proyective equivalences, get an expression that relates the scalar curvature $R$ and $\bar{R}$.

Solution Using [4],

$$
S_{i j}=\bar{S}_{i j}+\tau\left(F_{i j}-\bar{F}_{j i}\right),
$$


and as spaces of Einstein:

$$
S_{i j}=c_{1} g_{i j}, \quad \bar{S}_{i j}=c_{2} \bar{g}_{i j}
$$

$$
S_{i j}=\frac{R}{n} g_{i j}, \quad \bar{S}_{i j}=\frac{\bar{R}}{n} \bar{g}_{i j}
$$

it must be

$$
\frac{R}{n} g i j=\frac{\bar{R}}{n} \bar{g}_{i j}+\tau\left(F_{i j}-\bar{F}_{j i}\right), \quad \tau \in C,
$$

Applying $g^{i j}$ result

$$
\begin{gathered}
R=\frac{\bar{R}}{n} g^{i j} \bar{g}_{i j}+\tau\left(g^{i j} F_{i j}-g^{i j} \bar{F}_{j i}\right), \\
R=\frac{\bar{R}}{n} g^{i j} \bar{g}_{i j}+\tau\left(\|F\|-g^{i j} \bar{F}_{j i}\right)
\end{gathered}
$$

O

$$
R g_{i j}=\frac{\bar{R}}{n} \bar{g}_{i j}+\tau\left(\|F\| g_{i j}-\bar{F}_{i j}\right)
$$

Applying now $\bar{g}^{i j}$,

$$
R g_{i j}=\bar{R} \bar{g}_{i j}+\tau\left(\|F\| g_{i j}-\|\bar{F}\| \bar{g}_{i j}\right)
$$

from here

$$
(R-\tau\|F\|) g_{i j}=(\bar{R}-\tau\|\bar{F}\|) \bar{g}_{i j},
$$

then

$$
\frac{(R-\tau\|F\|)}{(\bar{R}-\tau\|\bar{F}\|)}=\frac{\operatorname{det}\left(\bar{g}_{i j}\right)}{\operatorname{det}\left(g_{i j}\right)}
$$

In [3] proof

$$
\ln \sqrt{\frac{\operatorname{det}\left(\bar{g}_{i j}\right)}{\operatorname{det}\left(g_{i j}\right)}}=(n+2) h, \quad h \in C^{\infty}(M) .
$$

Then he concludes

$$
(R-\tau\|F\|)=(\bar{R}-\tau\|\bar{F}\|) \exp [2 h(n+2)] .
$$

\section{Example}


Get an expression that compute the tensor Ricci in a compact kahlerian manifolds admitting proyective hollomorphic transformations with associated vector $F$, if $A_{n}$ this is recurrent.

Solution In this case

$$
\nabla_{k} R_{l j i}^{h}=R_{l j i}^{h} F_{k}
$$

by making the contraction $a=j$ and adding up from 1 to $n$ we obtain

$$
\nabla_{k} S_{j i}=S_{j i} F_{k}
$$

But

$$
\nabla_{k} S_{j i}=\partial_{k}\left(S_{j i}\right)-\Gamma_{k j}^{a} S_{a i}-\Gamma_{k i}^{a} S_{j a}
$$

Applying $g^{i j}$ result

$$
\nabla_{k} S_{j i}=\partial_{k}\left(S_{j i}\right)
$$

From (2.6) and (2.7) results

$$
\partial_{k}\left(S_{j i}\right)-S_{j i} \partial_{k} f=0 .
$$

The solution of this partial differential equation is the tensor de Ricci, hence is obtained scalar curvature.

\section{References}

[1] H. Izumi. A remark on infinitesimal holomorphically proyective transformations, Mat. Japonica, 39, pp. 47-60, (1994).

[2] H. Izumi, Y. Kazanari. On infinitesimal holomorphically proyective transformations in compact Kaehlerian manifolds, Hokkaido Math.J., 8, pp. 65-79, (1970).

[3] Malavé Guzmán R. Transformaciones holomorficamemte proyectivas equivalentes, Departamento e Matemáticas de la Universidad de Oriente, (tesis de maestria), (2007).

[4] R. Martínez, R. Ramírez. Lyra spaces. Their application to mechanics, Jadronic, J, 12, pp. 123-236, (1992). 


\section{Richard Malavé Guzmán}

Departamento de Electricidad,

Universidad Politécnica Territorial del oeste de

Sucre Clodosbaldo Russian, 6101 Cumaná, Edo. Sucre,

Venezuela

e-mail : rmalaveg@gmail.com

\section{Franmary López}

Departamento de Electricidad, Universidad Politécnica Territorial del oeste de Sucre Clodosbaldo Russian, 6101 Cumaná, Edo. Sucre, Venezuela

e-mail : franmalopezv@hotmail.com

and

\section{Rodrigo Martínez}

Departamento de Matemática, Universidad de Oriente, 6101 Cumaná, Edo. Sucre, Venezuela

e-mail : yigo54@cantv.net 